\title{
Limits on WIMP Dark Matter
}

\section{Neil Spooner* and Vitaly Kudryavtsev}

University of Sheffield, Dept. of Physics and Astronomy, Hounsfield Rd. Sheffield, S3 7RH, UK

in.spooner@sheffield.ac.uk, t. kudryavtsev@sheffield.ac.uk

m. spooner@sheffield.ac.ukg, vikudryavtsev@sheffield.ac.uk

ABSTRACT: The current state searches for dark matter in the form of Weakly Interacting Massive Particles (WIMPs) using both direct and indirect techniques is reviewed. Advances in recent years by various direct search experiments, utilising technology able to record the nuclear recoil events expected from elastic scattering by WIMPs, have allowed progress towards lower limits to be made. In particular, the Edelweiss and CDMS collaborations are achieving sensitiviy able to challenge data from DAMA interpreted as evidence for WIMPs of mass in the region of $60 \mathrm{GeV}$. Meanwhile, indirect searches, based on observing the annihilation products of neutralino-neutralino interactions in the Earth, Sun and Galaxy, have produced intriguing results. For instance, analysis by Superkamiokande now suggests limits comparable with the best direct search results.

\section{WIMP Direct Searches}

WIMPs interact with normal matter by elastic scattering off nuclei. The energy deposited by the resulting recoil nuclei or atoms has a characteristic exponential spectrum. This is determined mainly by the kinematics of the interaction, the WIMP mass relative to that of the recoiling nuclei and the velocity of the WIMP, determined by the velocity of the Earth through the galactic halo. The favoured range of WIMP masses, velocities and likely cross sections (for instance for MSSM) lead to recoil spectra expected to have energy ranging from a few $\mathrm{keV}$ upto a few hundred $\mathrm{keV}$ with rate $<1 \mathrm{~kg}^{-1} \mathrm{day}^{-1}$. The latter rate is typically a factor of $10^{6}$ lower than the ambient rate from background gammas due to surrounding natural radioactivity 剑.

These characteristics determine basic requirements of direct detection technology, the need for low energy threshold and some means of identifying genuine recoils from the much higher rate of background electron recoils. The latter is feasible in principle because the

\footnotetext{
${ }^{*}$ Speaker.
} 
energy loss per unit track length $(\mathrm{dE} / \mathrm{dx})$ for electrons is typically 10 times lower than for nuclear recoils [2] ray muons, can produce background nuclear recoils indistinguishable from those expected from WIMP interactions. Therefore, it is essential also that direct WIMP searches be performed in deep underground sites, typically $>1000$ mwe, where this flux is negligible or can be sufficiently reduced using neutron shielding.

Several technologies hold out prospects for achieving the requirements above but the most favoured at present are ionisation, scintillation and low temperature bolometric devices. Germanium ionisation detectors, used initially for double beta decay searches, set the first limits. More recently of note has been the Heidelberg-Moscow detector and the HDMS prototype Ge detectors [풀] operating at Gran Sasso. These have produced currently competitive limits. However, detectors using ionisation alone have no means of actively distinguishing nuclear recoils from electron background. Hence only limits can be set, based on the measured continuum background. The recent development of Ge detectors have thus tended to concentrate on material purification, in an effort to reduce intrinsic radioactivity. However, development towards larger mass Ge (10s-100s kg), exemplified by the GENIUS project [i] modulation of the dark matter event rate, arising from the earth's varying speed through the Galaxy.

Scintillation and low temperature detectors provide a route to the required additional

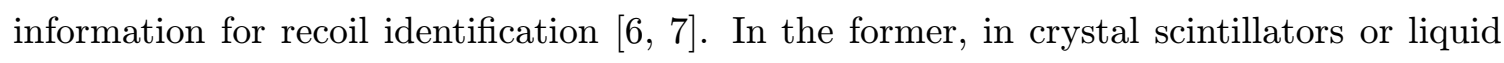
noble gases, the high $\mathrm{dE} / \mathrm{dx}$ for nuclear recoils results in pulse decay times a factor of 0.3 0.5 shorter than for electrons. Statistical analysis can then be used to identify a population

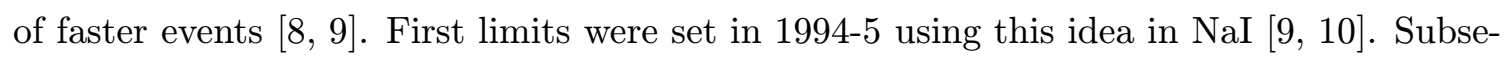
quently, following improved sensitivity, the UKDMC group at the Boulby site discovered a population of fast events at low energy in NaI, possibly due to surface alpha particles

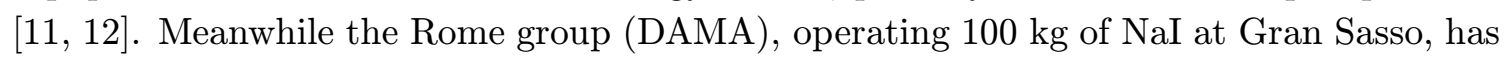
reported an annual fluctuation in the total count rate over 4 years. They interpret this as

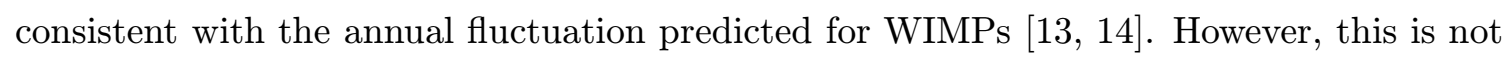
yet widely accepted because the technique does not separate nuclear recoils from the much larger low energy background which, in principle, could be subject to other modulating systematics [i] $\left.\overline{1}_{1} \overline{1}\right]$.

Several experiments based on counting total events in low temperature bolometers are underway and have set limits, notably by CRESST and the Milan group [1] '1] $1 \overline{1}$. However, of greater significance are schemes in which nuclear recoil identification is achieved in bolometric detectors by combining with simultaneous observation of ionisation or scintillation. The former is used by the CDMS-I and Edelweiss experiments, the latter

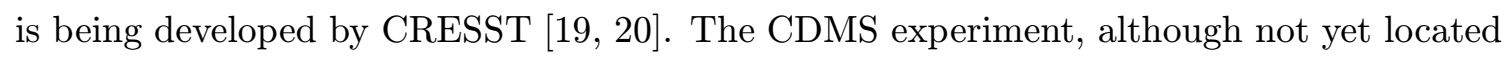
deep underground and hence needing to subtract neutron background, has presented data that appear to exclude the Rome result [2]1] limit around $2 \times 10^{-6} \mathrm{pb}$ in the mass range $20-100 \mathrm{GeV}$. Edelweiss have also released results that significantly cut into the Rome allowed region but with the advantage that no neutron

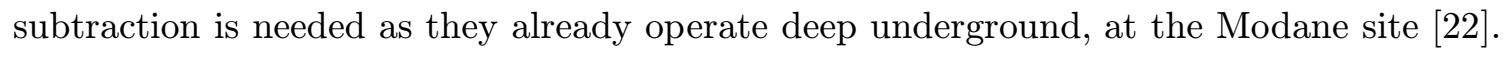


Recent results are summarised in Fig. 1, reproduced from [i2 $\overline{2} \overline{2}]$.

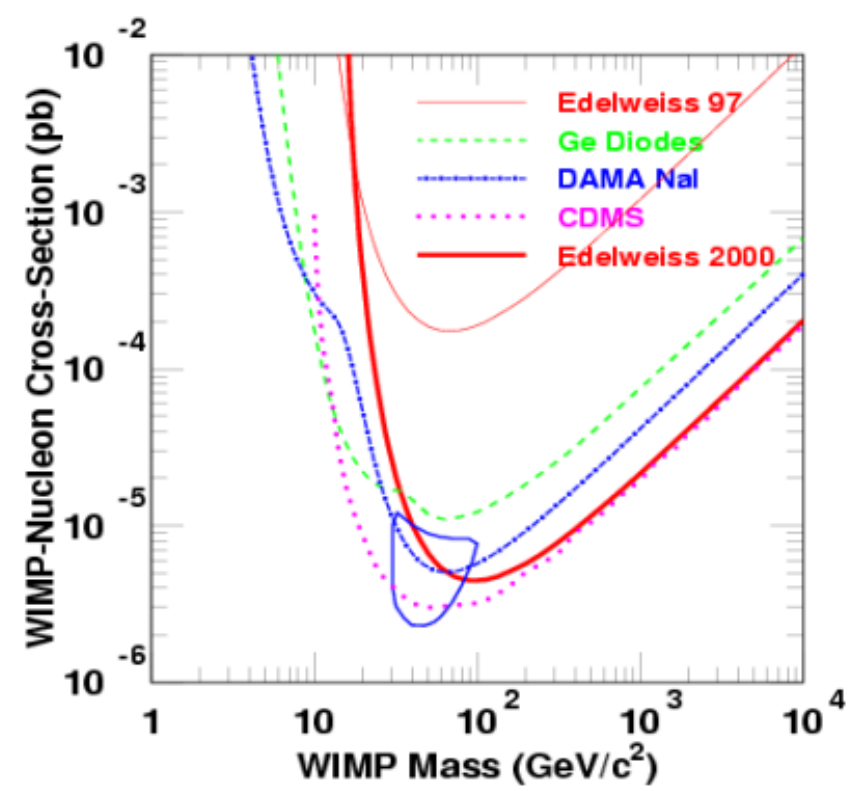

Figure 1: Recent results for spin independent WIMP searches.

New generations of experiment are being developed now aimed at factors of 10-1000 sensitivity improvements over 2-5 years. CDMS-II will be an expansion of the CDMSI experiment to be run in the Soudan Mine. CRESST-II, using scintillation plus low temperature technology at Gran Sasso is predicted to achieve similar sensitivity. Notable also is the growing interest in liquid Xe. Early experiments by the Rome group [203in] have now been supplemented by a Japanese group in Kamioka [2] $2 \overline{2} \overline{4}]$ ] and a major effort on xenon by the UKDM collaboration with UCLA, Torino, ITEP and Columbia [2. is constructing a series of liquid Xe experiments at Boulby. ZEPLIN I, now running at Boulby, is based on pulse shape discrimination. ZEPLIN II (see Fig. 2) makes use of simultaneous collection of scintillation and charge to achieve factors of 10-100 improved sensitivity and ZEPLIN III incorporates a high E-field in the liquid to enhance the recoil signal.

A $1000 \mathrm{~kg}$ liquid Xe detector, ZEPLIN-MAX, is currently being designed by the UKDM to achieve sensitivity below $<10^{-9}$ pb. Fig. 3 ilustrates the potential sensitivity of the liquid xenon experiments, estimated from preliminary results with ZEPLIN I.

Other novel techniques, in particular using superheated droplet detectors, may also eventually prove very sensitive $\left[\begin{array}{l}{[\overline{2}} \\ 2\end{array}, 1,2 \overline{2} \bar{T}_{1}\right]$ but ultimately the most convincing demonstration of the existence of WIMPs would be correlation of the direction of nuclear recoils with our motion through the Galaxy. The most promising technique to achieve this is by means of a low pressure Time Projection Chamber in which recoil tracks of a few $\mathrm{mm}$ length can be imaged. A UK/US collaboration is now running such a device of $1 \mathrm{~m}^{3}$ called DRIFT-I at Boulby [2] $\left.\overline{2}_{1}^{\prime}, \overline{2} \overline{2} \overline{9}\right]$. Fig. 4 shows preliminary underground Cf neutron calibration data for a short $43 \mathrm{~min}$ run from this detector taken with no passive shielding. Events are plotted 


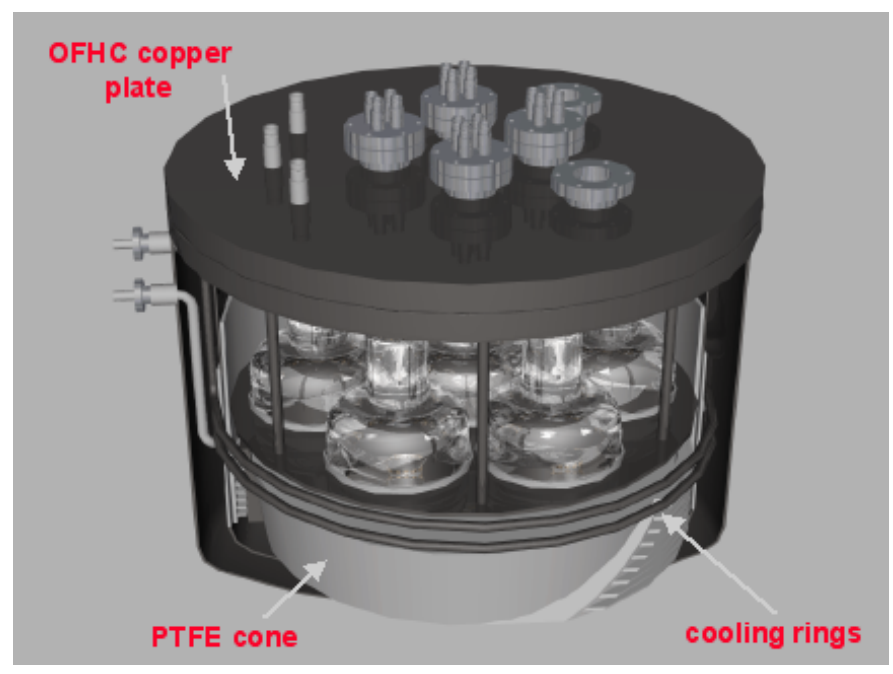

Figure 2: The ZEPLIN II liquid Xe detector of UKDM/UCLA/Torino.

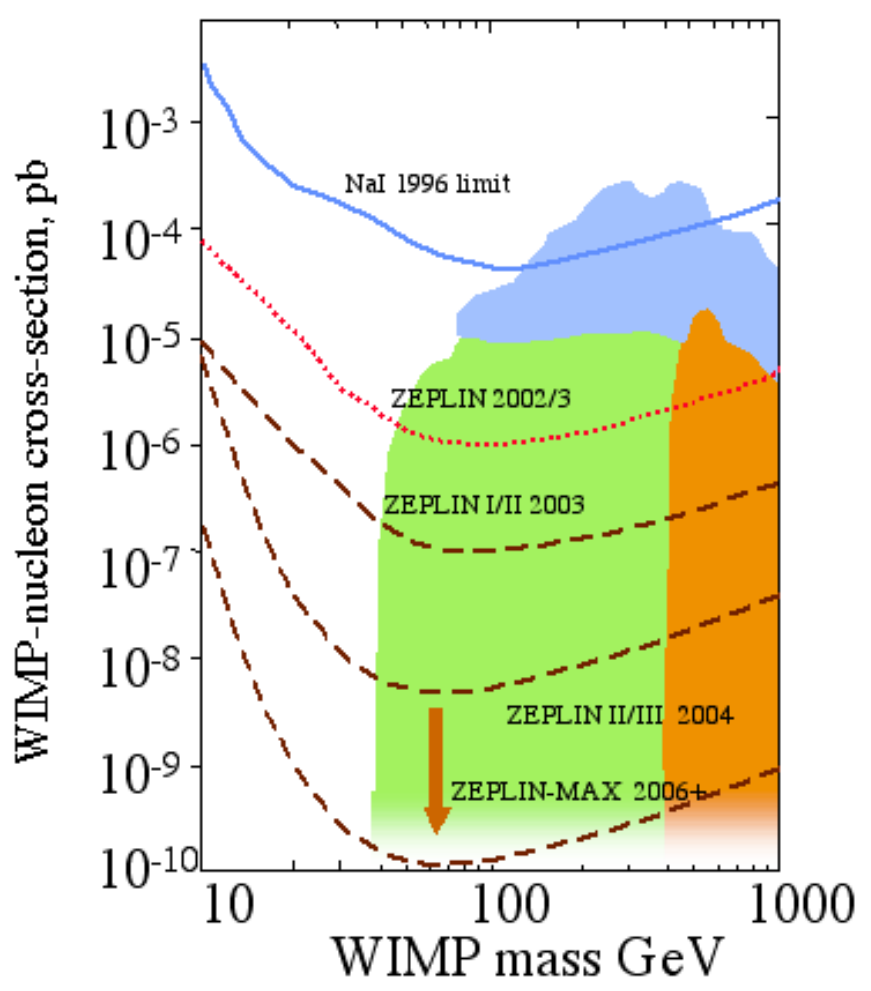

Figure 3: Predicted sensitivity of the ZEPLIN series of xenon dark matter detectors.

as number of ionizing pairs (NIPs) versus a discrimination parameter R2 that quantifies track range. Gammas are expected to show as events with relatively high R2, close to the vertical axis. However, sensitivity to gammas is so low that only neutron (nuclear recoil) events are observed, confirmed by runs without the neutron source. Such a directional dark matter detector offers the prospect of a dark matter "telescope" able to distinguish possible different velocity components of the dark matter that have been suggested could 
exist [i] $[\overline{0} \overline{0}]$.

\section{WIMP Indirect Searches}

If WIMPs are Majorana neutralinos then pair annihilations can occur and it may be possible to detect the resulting neutrinos, gamma rays, positrons or antiprotons. Such indirect detection of WIMPs is quite complementary to direct observation though much more model dependent, affected for instance by possible non-maxwellian velocity components in the halo. Indirect searches can be more sensitive to high mass WIMPs while neutralino models which produce low direct detection rates can sometimes produce substantial annihilation rates [ [3i $\left.\mathbf{1}_{1}^{1}\right]$, for example through the gamma-gamma channel. The most likely scenario is to search for high energy neutrino signals from the Sun, Earth, or galactic centre where the WIMP density may be sufficiently en-

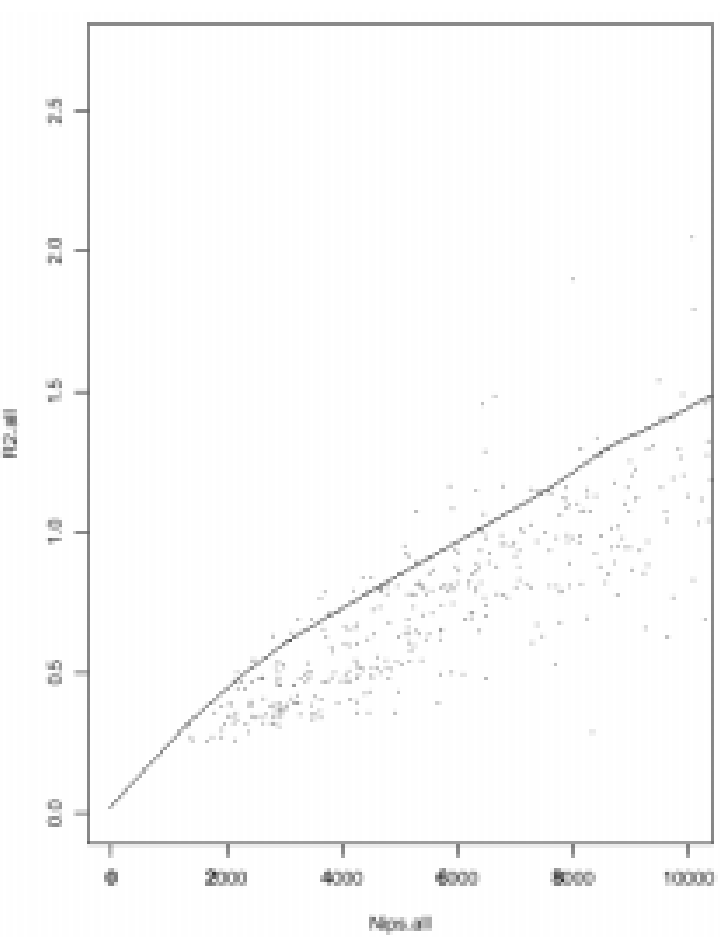

Figure 4: Neutron detection by the DRIFT-I directional dark matter detector. hanced by gravitational capture. The halo may provide a further source if the dark matter is clumpy [i] $\left.\overline{2}_{2}^{2}\right]$. Neutrinos, like annihilation gammas, have the advantage of maintaining their original direction.

Observation of muon neutrinos provide the best hope for the neutrino channel since the resulting upgoing muons produced in the Earth can be distinguished from background down-going atmospheric muons and have long range in present and planned Cherenkov neutrino detectors. These include AMANDA, ANTARES, IceCube, Baikal and NESTOR

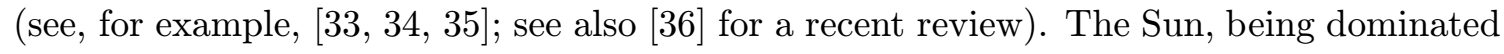
by hydrogen, is particularly favourable, with predictions of the muon rates for different neutralino models also easier to calculate. Nevertheless, calculations have been performed

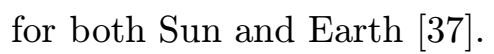

Present neutrino experiments have already provided significant limits on the Sun and Earth muon flux sufficient to constrain MSSM models [3 $3 \overline{8}, 3 \overline{3}$, $]$. Limits in the range $10^{3}-10^{4}$ muons $\mathrm{km}^{-2} \mathrm{yr}^{-1}$ are found for the Sun above $10^{2} \mathrm{GeV}$ and down to $10^{3}$ muons km${ }^{-2} \mathrm{yr}^{-1}$

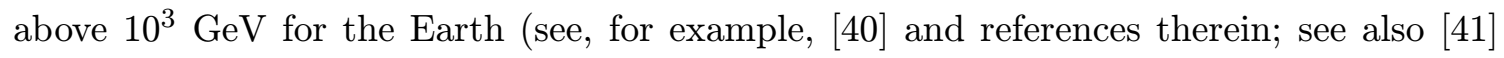
for a review). The latter is sufficient to indicate a possible contradiction with the DAMA direct search signal. A recent analysis by the SuperK collaboration to produce a WIMPnucleon cross section limit using combined Sun, Earth and galactic centre data (see Fig. 5) also appears to exclude parts of the DAMA allowed region [4]2i]. The AMANDA and ANTARES experiments are now aiming for $\mathrm{km}^{2}$ experiments that would provide a factor 
of $10^{4}$ improvement in sensitivity, sufficient to test large parts of the MSSM parameter

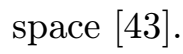

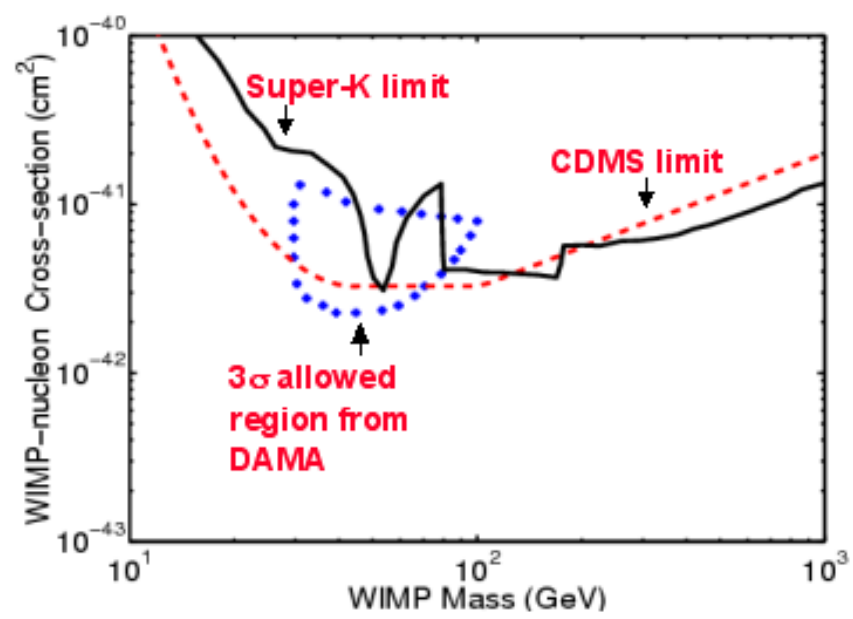

Figure 5: Combined Sun, Earth and galactic centre limit from SuperK [4는].

Searches for antiproton, positron and gamma ray lines from annihilation in the halo are also underway. The former two channels are hindered by uncertainty in galactic propagation models and the featureless nature of predicted spectra. Nevertheless balloon borne experiments to search for neutralino annihilation antiprotons at the top of the atmosphere

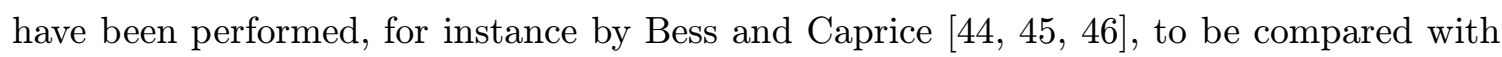
predictions of secondary antiproton background [īi]. The space experiment AMS aims also to undertake a search [4] cosmic-ray induced antiprotons, interesting limits can be placed for the highest annihilation rates [i] $[\overline{9} \overline{1}]$. Balloon observation of the positron continuum have also been performed. No excess over predictions from secondary positron production has been observed so far

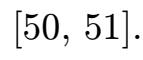

Although very sensitive to the local neutralino halo density, annihilation gamma-ray lines from the halo can be observed in principle by existing or planned Air Cherenkov Telescopes (ACTs) such as Veritas, Whipple, STACEE, CELESTE, MAGIC and MILAGRO, or by space-borne detectors including EGRET and GLAST. Indeed this technique may be the only one available to probe for heavy ( $\mathrm{TeV}$ ) stable neutralinos. The ACTs have acceptance angles suitable for searches of possible galactic centre signals. The high energy resolution of GLAST makes it suitable for high precision line searches. Recent MSSM calculations show that for a "standard" halo, for instance, Veritas and GLAST have discovery potential, with $\mathrm{TeV}$ masses accessible [1520,

\section{References}

[1] P. F. Smith, J.D. Lewin, Phys. Rep. 187 (1990) 203

[2] N. J. C. Spooner, Phys. Rep. 307 (1998) 253 
[3] H. V. Klapdor-Kleingrothaus et al., Proc. IDM2000, World Scientific, ed. N. Spooner and V. Kudryavtsev, York, UK (2000) 415

[4] H. V. Klapdor-Kleingrothaus et al., Proc. IDM2000, World Scientific, ed. N. Spooner and V. Kudryavtsev, York, UK (2000) 593

[5] S. Cebrian et al., Nucl. Phys. B (Proc. Suppl.) 95 (2001) 229

[6] N. J. C. Spooner et al., Phys. Lett. B 321 (1994) 156

[7] N. J. C. Spooner et al., Phys. Lett. B 273 (1991) 333

[8] P. Doll et al., Nucl. Instr. and Meth. in Phys. Res. A285 (1989) 464

[9] P. F. Smith et al., Phys. Lett. B379 (1996) 299

[10] R. Bernabei et al., Phys. Lett. B389 (1996) 757

[11] P. F. Smith et al., Phys. Rep. 307 (1998) 275

[12] V. A. Kudryavtsev et al., Phys. Lett. B452 (1999) 167

[13] R. Bernabei et al., Phys. Lett. B424 (1998) 195

[14] R. Bernabei et al., Nucl. Phys. B (Proc. Suppl.) 91 (2001) 361

[15] N. J. C. Spooner, Pub. Boston, Particles, Strings and Cosmology (1998) 130

[16] M. Altmann et al., Proc. 20th International Symposium on Lepton and Photon Interactions at High Energies (Lepton Photon 01), Rome, Italy, 23-28 Jul 2001 (astro-ph/0106314)

[17] A. Alessandrello et al., Nucl. Phys. B (Proc. Suppl.) 87 (2000) 78

[18] M. Vanzini et al., Nucl. Instr. and Meth. in Phys. Res. A461 (2001) 293

[19] N. E. Booth et al., Ann. Rev. Nucl. Part. Sci. 46 (1996) 471

[20] M. Bravin et al., Astropart. Phys. 12 (1999) 107

[21] R. Abusaidi et al., Phys. Rev. Lett. 84 (2000) 5699

[22] A. Benoit et al. Phys. Lett. B513 (2001) 15

[23] R. Bernabei et al., Phys. Lett. B436 (1998) 379

[24] Y. Suzuki, talk at the LowNu Workshop (Sudbury, Canada) (2000), hep-ph/0008296

[25] N. J. C. Spooner et al., Proc. DM 2000, Marina del Rey, ed. D. Cline (2000) 365

[26] N. Boukhira et al., Astropart. Phys. 14 (2000) 227

[27] J. I. Collar et al., New Jour. Phys. 2 (2000) 14

[28] M. J. Lehner et al., Proc. DARK98, Heidelberg, Germany, ed. H. V. Klapdor-Kleingrothaus (1998) 767

[29] C. J. Martoff et al., Nucl. Instr. and Meth. in Phys. Res. A440 (2000) 355

[30] M. Kamionkowski and A. Kinkhabwala, Phys. Rev. D57 (1998) 3256

[31] L. Bergstrom, Rept. Prog. Phys. 63 (2000) 793

[32] L. Bergstrom et al., Phys. Rev. D59 (1999) 043506

[33] E. Andres et al., Astropart. Phys. 13 (2000) 1 
[34] F. Montanet et al., Nucl. Phys. B (Proc. Suppl.) 87 (2000) 436

[35] V. A. Balkanov et al., Phys. of Atomic Nuclei, 63 (2000) 951

[36] F. Halzen, Phys. Rep. 333 (2000) 349

[37] L. Bergstrom, et al., Phys. Rev. D58 (1998) 103519

[38] M. M. Boliev et al., Nucl. Phys. B (Proc. Suppl.) 48 (1996) 83

[39] M. Ambrosio et al., Astrophys. J. 546 (2001) 1038

[40] X. Bai et al., Proc. IDM2000, World Scientific, ed. N. Spooner and V. Kudryavtsev, York, UK (2000) 499

[41] N. J. T. Smith, Proc. ICHEP2000, World Scientific, ed. C.S. Lim and T. Yamanaka, 1 (2000) 287

[42] A. Habig et al., Proc. 27th ICRC (Hamburg, Germany) HE3.05 (2001), hep-ex/0106024

[43] D. J. L. Bailey et al., Proc. 27th ICRC (Hamburg, Germany) HE3.05 (2001)

[44] A. Moiseev et al., Astrophys. J. 474 (1997) 479

[45] S. Orito et al., Phys. Rev. Lett. 84 (2000) 1078

[46] M. Boezio et al., Astrophys. J. 487 (1997) 415

[47] L. Bergstrom et al., Astrophys. J. 526 (1999) 215

[48] B. Alpat et al., Nucl. Instr. and Meth. in Phys. Res. A461 (2001) 272

[49] S. W. Barwick et al., Astrophys. J. 482 (1997) L191

[50] M. A. DuVernois et al., Astrophys. J., 559 (2001) 296

[51] M. Boezio et al., Proc. 26th ICRC (Salt Lake City, USA), 3 (1999) 57

[52] L. Bergsrom et al., Astropart. Phys. 9 (1998) 137

[53] Z. Bern et al., Phys. Lett. B411 (1997) 86 